
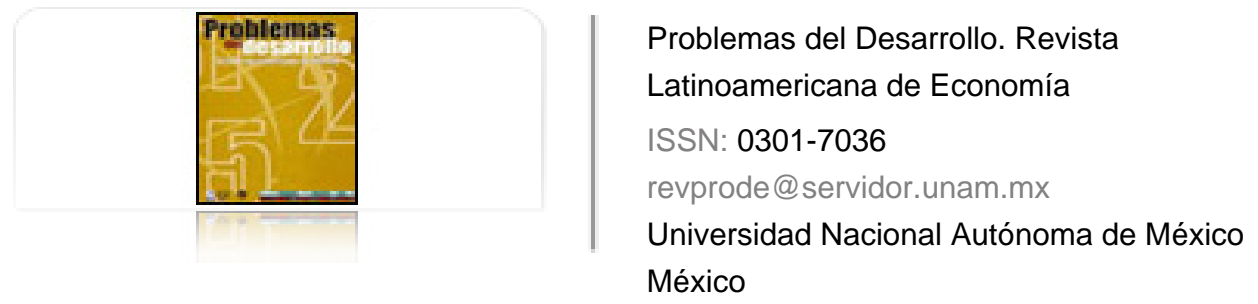

Palacios Hernández, Lylia; Ochoa Cortés, Arnoldo

Reestructuración y desindustrialización en la región sureste de Coahuila

Problemas del Desarrollo. Revista Latinoamericana de Economía, vol. 39, núm. 155, octubre-

diciembre, 2008, pp. 127-151

Universidad Nacional Autónoma de México

Distrito Federal, México

Disponible en: http://www.redalyc.org/articulo.oa?id=11820123007

- Cómo citar el artículo

- Número completo

- Más información del artículo

Página de la revista en redalyc.org

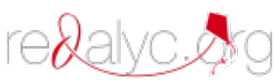

Sistema de Información Científica

Red de Revistas Científicas de América Latina, el Caribe, España y Portugal Proyecto académico sin fines de lucro, desarrollado bajo la iniciativa de acceso abierto 


\title{
REESTRUCTURACIÓN Y DESINDUSTRIALIZACIÓN EN LA REGIÓN SURESTE DE COAHUILA
}

\author{
Lylia Palacios Hernández* \\ Arnoldo Ochoa Cortés**
}

Fecha de recepción: 17 de junio de 2008. Fecha de aceptación: 24 de octubre de 2008.

\section{Resumen}

En este artículo se estudian las transformaciones en la industria manufacturera de la región sureste de Coahuila, resultado del ajuste estructural de los años ochenta en México, de la consolidación del modelo de apertura internacional, así como de la relocalización industrial que emprenden las corporaciones transnacionales hacia regiones periféricas, para mejorar su posición competitiva y su rentabilidad. Además se analizan los procesos característicos de la emergencia del régimen posfordista de acumulación y los cambios en la estructura manufacturera de la región y el comportamiento de actividades representativas, clasificadas por su desempeño por medio de indicadores como remuneración media, intensidad de capital, productividad laboral y rentabilidad de la fuerza de trabajo.

Palabras clave: sureste de Coahuila, industria, manufactura y reestructuración.

\section{Summary}

This article studies manufacturing industry transformations in the southeast region of the state of Coahuila. These are a result of structural adjustment in Mexico in the 1980s, of the consolidation of the international aperture model, and the industrial relocation in which the transnational corporations moved into the peripheral regions to improve their competitive position and their profitability. The paper also analyzes processes characteristic of the emergence of a post-Fordist system of accumulation, as well as changes in the manufacturing structure in the region and the performance of representative activities, classified by theirperformance, using such indicators as average remuneration, capital intensity, labor productivity and work force profitability.

Key words: southeast of Coahuila, industry, manufacturing and restructuring.

* Coordinadora del Área de Investigación en la Instituto de Investigaciones Sociales de la Universidad Autónoma de Nuevo León. Correo electrónico: lyliapalacios@yahoo.com.

** Profesor de tiempo completo en la Facultad de Economía. Universidad autónoma de Coahuila. Correo electrónico: ochoaarnoldo@ hotmail.com.

Este artículo se desprende de la tesis "Reestructuración productiva e inserción en la economía mundial: la industria manufacturera de la región sureste de Coahuila", que se desarrolla en el Programa de Doctorado en Ciencias Sociales con Orientación en Desarrollo Sustentable que ofrece la Universidad Autónoma de Nuevo León, con el reconocimiento del CONACYT como programa de calidad. Agradecemos los valiosos comentarios y sugerencias de los dictaminadores anónimos. 


\section{8}

LYLIA PALACIOS HERNÁNDEZ Y ARNOLDO OCHOA CORTÉS

\section{Résumé}

Dans cet article sont étudiées les transformations survenues dans l'industrie manufacturière de la région sud-est de l'état mexicain de Coahuila, qui sont le produit du réajustement structurel des années 80 au Mexique, de la consolidation du modèle d'ouverture internationale, ainsi que de la délocalisation de l'industrie vers les régions périphériques entreprise par les corporations transnationales pour améliorer compétitivité et rentabilité. De plus il est procédé à l'analyse des processus caractéristiques de l'émergence du régime post-fordiste d'accumulation, des changements survenus dans la structure manufacturière de la région et du comportement des activités représentatives, classifiées selon leur efficience estimée à partir d'indicateurs tels que la rémunération moyenne, l'intensité de capital, la productivité du travail et la rentabilité de la force de travail.

Mots clés: sud-est de l'état de Coahuila, industrie, manufacture et restructuration.

\section{Resumo}

Neste Artigo Estudam-se as mudanças na indústria da manufatura da região sudeste de Coahuila, como resultado do ajuste estrutural dos anos oitenta no México, da consolidação do modelo de apertura internacional além da relocalização industrial que impulsão as corporações internacionais com vistos em regiões periféricas para melhorar a posição competitiva e rentabilidade delas. Também se analisam os processos característicos da emergência do regime pós-fordista de acumulação e as mudanças na estrutura manufatureira da região e o comportamento de atividades representativas, classificadas pelo seu funcionamento mediante indicadores como remuneração média, intensidade de capital, produtividade laboral e rentabilidade da força de trabalho.

Palavras chave: sudeste de Coahuila, indústria, manufatura e reestruturação. 


\section{Introducción}

$\varepsilon$

n este trabajo se estudian las transformaciones en la industria manufacturera de la región sureste de Coahuila, integrada por los municipios de Saltillo,

Ramos Arizpe y Arteaga que conforman una zona conurbana, además de Parras y General Cepeda. Dichas transformaciones son resultado del ajuste estructural de los años ochenta y la consolidación del modelo de apertura internacional en México, así como de los procesos de relocalización industrial emprendidos por las corporaciones transnacionales para mejorar su posición competitiva y su rentabilidad, lo que constituye un rasgo característico de la emergencia del régimen posfordista de acumulación. El cambio de modelo significó para la región un fuerte crecimiento y una rápida inserción con la economía mundial mediante la exportación de manufacturas, principalmente asociada a la operación de las empresas relocalizadas en la región.

De inicio se exponen los cambios en la estructura industrial y se analizan las transformaciones en ramas de actividad representativas de la región. A partir de lo anterior se identifican, mediante una propuesta de clasificación, las actividades manufactureras que logran mantener o mejorar su ubicación regional, así como aquellas que perdieron presencia en el escenario industrial de la región. En ese sentido, se analizan las transformaciones generadas por las actividades manufactureras con base en la evolución de indicadores como remuneración media, intensidad de capital, productividad laboral y rentabilidad de la fuerza de trabajo.

\section{Marco conceptual: la transición posfordista}

El régimen de acumulación fordista permitió un periodo de crecimiento sostenido, en especial después de la Segunda Guerra Mundial, sobre la base de un patrón productivo sustentado en la mecanización de la producción y la segmentación técnica del trabajo, así como en una evolución que procuraba indexar la productividad, con los salarios y el costo de la vida, para permitir que la demanda se expandiera de manera paralela a la producción. Sin embargo, la economía mundial presentó una severa crisis en la década de los setenta del siglo XX, considerada por la Escuela de la Regulación como el resultado del agotamiento de dicho régimen de acumulación. A partir de entonces se inició un proceso de reestructuración con repercusiones sobre la vida económica y social del mundo (Leborgne y Lipietz, 1992; Aglietta, 1979; Gutiérrez Garza, 2003). 
La crisis del fordismo cuestionó el costo del Estado benefactor y los efectos inflacionarios del gasto público ejercido bajo criterios de corte keynesiano (Aglietta, 1979). A la par, emergieron formas flexibles de producción que fueron capaces de dar solución a la crisis de rentabilidad industrial derivada de la rigidez fordista, aunada a nuevas formas de organización del trabajo y redefinición de las relaciones laborales (Coriat, 1992; Schonberger, 1987). De forma adicional, el proceso prevé la utilización de tecnologías asociadas a la informática y las telecomunicaciones, una transformación de la división internacional del trabajo, menor intervención directa del Estado en la economía y una menor regulación de los procesos económicos, así como la emergencia del neoliberalismo como paradigma teórico dominante. Ello permitió reencauzar la economía mundial en el crecimiento sobre las bases de un nuevo régimen de acumulación denominado por los regulacionistas como posfordista.

El posfordismo muestra cómo una de sus características, la desconcentración técnica de la producción, que se observa en la relocalización productiva que rebasa las fronteras nacionales, repercute en la redefinición de la división internacional del trabajo. La relocalización de procesos o segmentos de la producción posibilitó eludir el costo salarial que en las economías centrales erosionó la ganancia.

En relación con las formas de integración de las regiones económicamente atrasadas bajo al proceso de globalización, Lipietz (1997) menciona una de ellas y la denomina como taylorización primitiva. Consiste en la dislocación de segmentos de la producción industrial que se trasladan hacia regiones con tasas de explotación muy elevadas, con una composición técnica del capital baja, lo que permite asociarlas con la industria maquiladora. Asimismo, la dinámica de la producción industrial en años recientes permite distinguir actividades de alto nivel tecnológico que se trasladan a regiones periféricas para establecer formas de organización productiva que corresponden a la producción flexible.

El deterioro del empleo y el salario ha sido característico del patrón de acumulación posfordista (Sotelo, 2002; Abramo, 2001) y se remonta al comienzo de la reestructuración productiva, manifestándose una tendencia al incremento del desempleo en los países industrializados (Rendón, 1999).

\section{Apertura internacional y crecimiento industrial en el sureste de Coahuila}

El periodo de vigencia del régimen fordista de acumulación corresponde en México al modelo de industrialización por sustitución de importaciones, que retomó las 
definiciones teóricas del keynesianismo para la definición de la política económica, al mismo tiempo que se asume la propuesta estructuralista emanada de la Comisión Económica para América Latina y el Caribe (CEPAL) para resolver los problemas estructurales (Soria, 2000; Aboites, 1989).

Una vez que la crisis de los años ochenta marcó el inicio de la transición mexicana hacia un modelo de apertura internacional, en el país se observan transformaciones encaminadas a adecuar las estructuras sociales y económicas a las condiciones marcadas por la emergencia del régimen de acumulación posfordista en las economías centrales.

Lo anterior generó cambios no sólo en las estructuras productivas, sino también en los mecanismos de integración de las regiones del mundo a una división internacional del trabajo que también se redefinía de acuerdo con las necesidades de acumulación capitalista (Valenzuela, 1988; Sotelo, 1988). En el caso de México, se modifica la estructura del comercio exterior al impulsar las exportaciones de productos industrializados (Mariña 2004; Basave, 2002; Gutiérrez Garza, 2004). Esta reorientación de la producción manufacturera ha sido acompañada por el fenómeno de relocalización productiva arriba mencionado.

En el estado de Coahuila, el cambio de modelo económico transformó el perfil productivo y la distribución territorial de la industria. Durante la vigencia de la política de industrialización sustitutiva de importaciones, la mayor parte de la producción manufacturera se generaba en la ciudad de Monclova, en torno a la industria siderúrgica apuntalada en la empresa paraestatal Altos Hornos de México S. A (Gutiérrez, 1993; Meléndez 1994; Cárdenas y Redonnet, 1998).

Sin embargo, una vez que se transita al modelo de apertura internacional, el espacio de mayor dinamismo se ubicó en la región sureste, espacio en el cual bajo el modelo de economía cerrada se logró constituir una estructura manufacturera relativamente diversificada, aunque con una especialización en torno a la industria metalmecánica (De Bell, 2005). Con lo anterior, el perfil productivo de Coahuila habría de transitar de la producción de un insumo trascendental para el desarrollo de la industria nacional, como fue el acero, hacia la producción de bienes de consumo duradero para el mercado mundial como automóviles, motores y autopartes, correspondiendo con la división internacional del trabajo asociada a los nuevos patrones de acumulación en México.

En una tendencia diferente al pobre desempeño de la economía mexicana durante los años ochenta, la región sureste de Coahuila presenta una tasa media de crecimiento industrial de $21.9 \%$ anual en la producción y de $8.1 \%$ en el empleo en-

Vol. 39, núm. 155, octubre-diciembre / 2008

$\underset{\text { DeSAarrollo }}{\text { PRR DEL MAS DEL }}$ 
tre 1980 y 1988, datos que en sí mismos denotan un estilo de crecimiento intensivo en capital y de alta productividad. Sin embargo, este crecimiento manufacturero no se manifestó de manera homogénea en las diversas ramas manufactureras, ya que fueron las actividades productoras de bienes duraderos y de capital las que imprimieron la dinámica y de manera muy particular la industria automotriz, que desde los primeros años de la transición estructural apuntaba a convertirse en la actividad más importante de la región. Con la apertura y desregulación económicas, el sureste de Coahuila aumentó su participación en la producción industrial de 23\% que tenía en 1980 a 61.8\% en 2003 [Instituto Nacional de Estadística Geografía e Informática (INEGI), 1988; 2005].

Bajo el modelo de economía abierta, las regiones ubicadas en el norte de México han tenido una fuerte participación en la captación de inversión tanto nacional como extranjera, así como en la participación en las exportaciones (Dávila, 1994). Actividades que se localizan principalmente en ciudades intermedias cercanas a la frontera, como Saltillo y Ramos Arizpe (Calderón y Martínez, 2004).

La distribución regional de la inversión privada total en Coahuila entre diciembre de 1999 y marzo de 2004 fue de 4,568.97 millones de dólares, de los cuales 69.7\% se invirtió en la zona sureste, dato congruente con la mayor dinámica manufacturera que ha mostrado la región a raíz de la apertura económica. En el periodo mencionado, dicha inversión logró crear 75,701 empleos en la manufactura, de los cuales 28.6\% se generó en el sureste.

Estos porcentajes de participación — tanto de la inversión canalizada como de los empleos generados - revelan que en la región sureste el porcentaje de inversión es muy superior al del empleo que produce, relación que permite afirmar que la inversión en la región sureste se destina a procesos productivos intensivos en capital.

De manera más específica y de acuerdo con la información de la Secretaría de Economía, 35.4\% de las empresas con inversión extranjera en 2006 se localiza en los municipios de Saltillo y Ramos Arizpe y del total de la inversión extranjera directa (IED) canalizada a la entidad entre 1999 y 2006, 28.5\% correspondió a la industria automotriz, lo que confirma una tendencia observada desde los años noventa (Mendoza, 2001).

En lo que se refiere al comercio exterior, a finales de la década de los ochenta la estructura de exportaciones de Coahuila ya descansaba sustancialmente en la industria automotriz, con poco más de dos terceras partes del total exportado por la entidad en términos de valor, actividad localizada sobre todo en la región sureste. 
Ya para 1996, con $88 \%$ de las principales empresas exportadoras, la región sureste participó con cerca de $77 \%$ del valor exportado por compañías no maquiladoras en la entidad. De los 13 productos manufactureros con mayor presencia en el comercio exterior de Coahuila, aparecen 11 cuyo origen se ubica sobre todo en la región sureste: vehículos, motores para automóvil, autopartes, textiles, productos metálicos, productos químicos y farmacéuticos, papel higiénico, sanitarios, loseta de barro y cerámica, piezas de peltre para cocina y alimentos procesados. En el mismo trabajo se señala que $88 \%$ de las principales empresas exportadoras se encontraban en la región sureste (Dávila, 1998).

En la región sureste de Coahuila la industria maquiladora cobró importancia a partir de la segunda mitad de la década de ochenta, cuando este tipo de producción inició un proceso de expansión hacia municipios no fronterizos. Sin embargo, las empresas maquiladoras en esta región no se han constituido como el eje principal de la industrialización, como sí ocurre en la región de la frontera (Martínez, 1993).

\section{Transformación de la estructura manufacturera}

Desde los últimos años de vigencia en México del modelo orientado al mercado interno, la estructura manufacturera de la región sureste de Coahuila manifiesta una especialización productiva en torno a las ramas generadoras de bienes duraderos y de capital, mismas que representaron 59\% del valor de la producción manufacturera de la región en 1980. Sólo dos actividades generadoras de bienes perecederos alcanzan importancia: alimentos y textil, y sólo una productora de insumos, la de productos de minerales no metálicos (cuadro 1).

En los años correspondientes al ajuste estructural de los ochenta se consolidó la rama de equipo de transporte como la más relevante y la que marcaría el ritmo del crecimiento manufacturero en la región, su presencia se incrementó de 4.6\% en 1980 a 72.2\% en 1988. En esta actividad se concretaron procesos de relocalización de corporaciones automotrices de capital estadounidense que buscaron, mediante la instalación de plantas en regiones como la que estudiamos, restituir la rentabilidad y mejorar su posición competitiva para hacer frente al embate de las armadoras orientales en el mercado mundial.

Una vez que se consolida el modelo de apertura internacional en la economía mexicana y se recupera el crecimiento, la manufactura en el sureste de Coahuila mantiene su dinámica, aunque a un ritmo menor al mostrado en el periodo de ajuste de los años ochenta; la tasa media de crecimiento del producto entre 1988 y 2003 fue

Vol. 39, núm. 155, octubre-diciembre / 2008

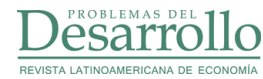




\begin{tabular}{|c|c|c|c|c|c|c|c|c|}
\hline Estructura de la in & ndustri & $\begin{array}{l}\text { Región } \\
\text { manufa }\end{array}$ & $\begin{array}{l}\text { Cuadro } \\
\text { sureste d } \\
\text { cturera }\end{array}$ & $\begin{array}{l}1 \\
\text { e Coahu } \\
{[1980,19}\end{array}$ & 1998,2 & 03 (por & ntajes)] & \\
\hline & & Produc & cto bruto & & & Persona & ocupado & \\
\hline & 1980 & 1988 & 1998 & 2003 & 1980 & 1988 & 1998 & 2003 \\
\hline No duraderos & 24.1 & 8.0 & 12.1 & 6.6 & 26.2 & 27.6 & 36.5 & 32.3 \\
\hline Alimentos & 6.2 & 4.0 & 4.6 & 3.8 & 4.9 & 9.3 & 13.0 & 16.6 \\
\hline Bebidas & 2.2 & 0.6 & 1.1 & 0.8 & 0.7 & 1.8 & 2.4 & 2.4 \\
\hline Tabaco & - & - & - & - & - & $\cdot$ & - & - \\
\hline Textil & 12.0 & 2.4 & 4.8 & 1.1 & 12.7 & 6.7 & 6.6 & 4.7 \\
\hline Vestido & 2.1 & 0.5 & 0.5 & 0.3 & 3.8 & 4.5 & 8.1 & 3.7 \\
\hline Cuero y calzado & 0.1 & n.s. & 0.4 & 0.1 & 0.2 & 0.1 & 0.7 & 0.6 \\
\hline Madera y corcho & 0.2 & 0.1 & 0.1 & 0.1 & 0.4 & 0.9 & 0.8 & 0.6 \\
\hline Muebles de madera & 0.1 & 0.1 & 0.3 & 0.1 & 0.7 & 1.6 & 2.2 & 1.8 \\
\hline Editorial e imprenta & 0.2 & 0.2 & 0.1 & 0.1 & 1.2 & 1.8 & 1.4 & 1.1 \\
\hline Otras manufacturas & 1.0 & 0.1 & 0.2 & 0.1 & 1.6 & 0.9 & 1.3 & 0.8 \\
\hline Intermedios & 16.8 & 12.4 & 11.8 & 12.6 & 15.1 & 21.9 & 12.9 & 19.5 \\
\hline Papel & - & n.s. & 1.0 & 2.5 & - & n.s. & 1.2 & 2.0 \\
\hline Hule y plástico & 0.8 & 0.3 & 2.0 & 2.3 & 1.0 & 1.3 & 1.9 & 5.2 \\
\hline Química & 1.6 & 2.9 & 3.6 & 3.0 & 2.1 & 4.2 & 2.8 & 4.0 \\
\hline Derivados de carbón y petróleo & - & n.s. & 0.1 & - & - & n.s. & 0.1 & 0.1 \\
\hline Productos de minerales no metálicos & s 14.2 & 5.1 & 4.9 & 3.4 & 11.9 & 10.9 & 6.6 & 5.0 \\
\hline Metálica básica & 0.2 & 4.1 & 0.2 & 1.4 & 0.1 & 5.5 & 0.3 & 3.2 \\
\hline Duraderos y de capital & 59.1 & 79.6 & 76.0 & 80.9 & 58.7 & 50.2 & 50.3 & 48.1 \\
\hline Productos metálicos & 33.4 & 2.3 & 3.3 & 1.0 & 39.6 & 11.0 & 13.6 & 9.6 \\
\hline Maquinaria y equipo no eléctrico & 19.5 & 3.8 & 4.2 & 1.3 & 9.4 & 4.3 & 5.5 & 4.3 \\
\hline $\begin{array}{l}\text { Maquinaria y equipo eléctrico } \\
\text { y electrónico }\end{array}$ & 1.6 & 1.3 & 3.2 & 0.1 & 4.3 & 5.8 & 14.9 & 0.8 \\
\hline Equipo de transporte & 4.6 & 72.2 & 65.3 & 78.5 & 5.4 & 29.1 & 16.3 & 33.4 \\
\hline Total & 100.0 & 100.0 & 1000 & 1000 & 100.0 & 100.0 & 100.0 & 100.0 \\
\hline
\end{tabular}

Fuente: INEGI, XI Censo Industrial, 1981. INEGI, XIII Censo Industrial, 1989. INEGI, XV Censo Industrial, 1999. INEGI, XVI Censo Industrial, 2004.

de $4.2 \%$, en tanto la generación de empleos se redujo a $1.6 \%$ anual en promedio, lo que pone en evidencia un crecimiento con escasa capacidad generadora de empleo.

Después del ajuste de los años ochenta las actividades productoras de bienes de consumo perecedero disminuyen su participación, entre éstas únicamente la industria de alimentos mantiene una presencia significativa. Las ramas generadoras de insumos también disminuyen su participación porcentual, sólo las industrias química y de productos de minerales no metálicos conservan una aportación superior a 3\%. 
Por su parte, las ramas productoras de bienes duraderos y de capital elevan aún más su contribución hasta llegar a $80.9 \%$ en 2003, lo que acentúa la especialización regional en torno a estas actividades. Al interior de este grupo, la rama de equipo de transporte representó $78.5 \%$ de la producción manufacturera de la región, lo que agudiza una concentración industrial que también se manifiesta en la estructura del comercio exterior y en la captación de inversión extranjera (Dávila, 1998; Martínez, 2005).

\section{Reestructuración y desindustrialización}

La clasificación de las actividades que presentamos — ramas en reestructuración; reestructuración no lograda y actividades en desindustrialización — se desprende del carácter heterogéneo del proceso. Por tal motivo, con información censal se construyen indicadores que nos acercan a los conceptos de remuneración media, productividad laboral, intensidad de capital y rentabilidad del uso de la fuerza de trabajo. ${ }^{1}$ En ese sentido, consideramos que un desempeño favorable en los indicadores significa que las actividades lograron una reestructuración que les permitió una mejor ubicación en la región, en caso contrario el intento no fue exitoso o no se inició.

\section{Ramas que se reestructuran}

Entre las actividades que logran reestructurarse, para mantener o incrementar un lugar en la manufactura de la región, ubicamos a las ramas productoras de equipo de transporte, alimentos, industria química y de bienes de minerales no metálicos. Una primera característica de estas actividades es el sostenimiento de tasas positivas de crecimiento

1 Las remuneraciones promedio recibidas por la fuerza de trabajo fueron calculadas con la división de las remuneraciones totales al personal ocupado entre el número de trabajadores en la respectiva rama de actividad para los años censales considerados.

La productividad laboral será entendida como el valor generado por un trabajador en el año censal y por tanto es un cociente entre valor agregado y el número de trabajadores de la rama respectiva, mientras que la intensidad de capital es la relación de activos fijos por trabajador, es decir, el cociente entre activos fijos netos y el personal ocupado.

Rentabilidad por el uso de la fuerza de trabajo es un cociente entre el valor promedio generado y la remuneración media que recibe; con ello nos aproximaremos a comprender en qué medida el uso de la mano de obra en la región es rentable para las empresas, por el valor que genera respecto a lo que el capitalista paga por su utilización en el proceso productivo. Un indicador de uno significa que la totalidad del valor generado por el trabajador se paga en remuneraciones y por tanto no hay una ganancia para el capital por el uso de la fuerza de trabajo; si dicho indicador es superior a uno, el uso de fuerza de trabajo es rentable puesto que el trabajador promedio genera un valor superior a lo que recibe.

Vol. 39, núm. 155, octubre-diciembre / 2008

$\underset{\text { DeSarrollo }}{\text { PRR }}$ 
tanto en producción como en empleo a lo largo del periodo de estudio, con excepción de minerales no metálicos que reduce su nivel de empleo entre 1988 y 2003.

A continuación presentamos el comportamiento particular de cada una de dichas actividades mediante la evolución de los indicadores mencionados.

Equipo de transporte

La rama de equipo de transporte, como hemos reiterado, se convierte desde la década de los ochenta en la más trascendente en el sureste de Coahuila. Aumenta su participación productiva de $4.6 \%$ en 1980 a 78.5 en 2003 , y de 5.4 a $33.4 \%$ en el empleo en el mismo periodo, por lo que es la rama más fortalecida por la transición de la economía mexicana a un modelo de apertura internacional.

El crecimiento de la rama es más acelerado en los años de transición al modelo de apertura internacional con $72 \%$ en producción y 33\% en empleo entre 1980 y 1988, para luego moderar su ritmo de crecimiento a 4.8\% en producción entre 1988 y 2003, al mismo tiempo que se reduce su capacidad generadora de empleo a $2.5 \%$ anual.

Las empresas que detonaron el crecimiento de la rama automotriz fueron las transnacionales General Motors y Chrysler que, de acuerdo con la estrategia de relocalización, se establecen en el municipio de Ramos Arizpe al comenzar su operación en 1982, para dar inicio a un proceso de articulación de empresas del ramo, bajo esquemas de calidad total y justo a tiempo, característicos de la producción flexible (Micheli, 1994; Ramírez, 1999). La producción de estas empresas estaría enfocada desde el inicio al abastecimiento del mercado externo (Dávila, 1982; Arteaga, 1988; Arteaga, 1985). Por lo anterior, se puede hablar de la construcción del cluster más dinámico de la región (Dávila, s/f).

Sin olvidar la fuerte y generalizada caída de la remuneración promedio de los trabajadores a consecuencia del ajuste estructural de los años ochenta, la rama de equipo de transporte a lo largo del periodo analizado ha sostenido indicadores de remuneración media superiores al promedio manufacturero regional (cuadro 2).

En cuanto a la productividad laboral de la rama, se observa un crecimiento muy importante, debido a las fuertes inversiones realizadas en la región para iniciar la construcción del complejo automotor e introducir procesos de producción basados en tecnología flexible. Como consecuencia de las inversiones mencionadas, la actividad se convirtió en los años ochenta en la más intensiva en capital.

Después del ajuste estructural, la rama inició un proceso constante y pronunciado de reducción en la productividad laboral que, por otro lado, se acompañó de un au- 
Cuadro 2

Rama de equipo de transporte

Indicadores de desempeño $(1980,1988,1998$ y 2003)

\begin{tabular}{lrrrrr}
\hline & 1980 & 1988 & 1998 & 2003 & Variación 1980-2003 \\
\hline Remuneración media & 44.06 & 35.09 & 38.69 & 43.19 & $(0.1)$ \\
Productividad laboral & 75.75 & 622.50 & 332.44 & 231.74 & 205.9 \\
Intensidad de capital & 63.59 & 363.70 & 433.25 & 525.28 & 726.0 \\
Rentabilidad de fuerza de trabajo & 1.72 & 17.74 & 8.59 & 5.36 & 211.6 \\
\hline
\end{tabular}

Nota: Cifras en miles de pesos de 1973. En el Censo Industrial de 1980 no se presenta información sobre activos fijos, el indicador para dicho año se construyó con datos de 1975.

Fuente: SPP, X Censo Industrial, 1976. INEGI, XI Censo Industrial, 1981. INEGI, XIII Censo Industrial, 1989. INEGI, XV Censo Industrial, 1999. INEGI, XVI Censo Industrial 2004.

mento en la intensidad de capital. La reducción en productividad puede asociarse a la conformación de un complejo automotor cada vez más amplio, que paulatinamente integra a empresas con diversos niveles tecnológicos que se establecen en la región para integrarse en la cadena de suministros de las grandes armadoras mediante mecanismos de subcontratación.

Durante los años ochenta y como resultado del aumento en productividad y la reducción de las remuneraciones, la rama manifestó un explosivo incremento en su rentabilidad, la que se multiplicó por 10 entre 1980 y 1988. A partir de ese año, se observa una tendencia a la reducción del indicador, explicable en los años de consolidación del modelo de apertura internacional, por la evolución descendente de la productividad y ascendente de la remuneración media.

El comportamiento de los indicadores durante el periodo de análisis en su totalidad muestra el fuerte proceso de transformación experimentado por la rama, lo que la convierte en el referente en cuanto a reestructuración.

\section{Industria de alimentos}

La producción de alimentos se mantiene como una actividad importante en la estructura manufacturera del sureste de Coahuila. Aunque su participación se redujo en los años del ajuste estructural, por el vertiginoso crecimiento de equipo de transporte. La industria de alimentos creció a una tasa media superior a 15\% en la producción y a más de 17\% en el empleo. Posteriormente, entre 1988 y 2003 los ritmos de crecimiento de producción y empleo se redujeron a $3.8 \%$ y $5.6 \%$ en cada caso.

A lo largo del periodo estudiado, la rama de alimentos ha sido una de las actividades de más baja remuneración e invariablemente se ha ubicado por debajo del prome- 
dio regional. Por otro lado, desde 1980 hasta 2003 un proceso sostenido de aumento en la productividad laboral le permitió alcanzar un indicador superior al promedio regional en este aspecto (cuadro 3).

Cuadro 3

Rama de alimentos

Indicadores de desempeño (1980, 1988, 1998 y 2003)

\begin{tabular}{lrrrcc}
\hline & 1980 & 1988 & 1998 & 2003 & Variación 1980-2003 \\
\hline Remuneración media & 18.43 & 15.10 & 21.19 & 16.18 & $(12.2)$ \\
Productividad laboral & 48.36 & 75.24 & 79.43 & 86.48 & 78.8 \\
Intensidad de capital & 33.87 & 50.94 & 50.82 & 33.03 & $(2.5)$ \\
Rentabilidad de fuerza de trabajo & 2.62 & 4.98 & 3.75 & 5.34 & 103.8 \\
\hline
\end{tabular}

Nota: Cifras en miles de pesos de 1973. En el Censo Industrial de 1980 no se presenta información sobre activos fijos, el indicador para dicho año se construyó con datos de 1975.

Fuente: SPP, X Censo Industrial, 1976. INEGI, XI Censo Industrial, 1981. INEGI, XIII Censo Industrial, 1989. INEGI, XV Censo Industrial, 1999. INEGI, XVI Censo Industrial, 2004.

Como resultado del aumento constante de la productividad y la persistencia de bajos niveles de remuneración, la rama muestra en el periodo de análisis una tendencia ascendente en la rentabilidad derivada del uso de la fuerza de trabajo, para ubicarse en 2003 en un nivel superior al promedio de la región y muy cerca del registrado por la rama más representativa que es la del equipo de transporte.

En este caso, podemos señalar que la reestructuración de la industria de alimentos se ha sustentado en una limitada inversión en medios de producción que se manifiesta en una baja intensidad de capital, que es característica en actividades generadoras de bienes de consumo perecedero. Sin embargo, la rama ha mostrado aumentos en la productividad laboral derivada de una mayor eficiencia en la organización productiva de los trabajadores, que se mantienen con baja remuneración a pesar de incrementar su productividad.

\section{Industria química}

La generación de productos químicos es una actividad que crece en la región, sobre todo con la apertura y la desregulación económicas. El incremento más significativo se presentó durante los años del ajuste estructural, ya que entre 1980 y 1988 mostró tasas medias de crecimiento de $31.3 \%$ y $17.9 \%$ en producción y empleo. Entre 1988 y 2003 el aumento promedio de la producción se ubicó en $4.5 \%$ anual mientras que el empleo sólo creció $1.3 \%$.

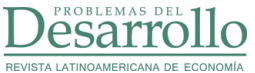


A lo largo del periodo de estudio, la industria química presenta un aumento en las remuneraciones promedio pagadas al personal ocupado, por lo que, según los datos más recientes, paga los sueldos y salarios medios más altos de la región (cuadro 4).

Esta rama es una actividad que ha presentado fuertes incrementos en productividad bajo el modelo de apertura internacional. Además, el comportamiento de la intensidad de capital guarda un paralelismo con la productividad, lo que se explica sobre todo por el arribo de empresas de capital transnacional como Fersinsa Gist Brocade y Perrigo, esta última estableció en los años recientes dos plantas para elaboración y empacado de medicamentos, además de adquirir una empresa de amplia tradición en la región como Química y Farmacia. Los procesos de producción en estas firmas son altamente automatizados.

Cuadro 4

Industria química

Indicadores de desempeño (1980, 1988, 1998 y 2003)

\begin{tabular}{lrrrrc}
\hline & 1980 & 1988 & 1998 & 2003 & Variación 1980-2003 \\
\hline Remuneración media & 22.26 & 26.56 & 53.41 & 49.82 & 123.8 \\
Productividad laboral & 41.94 & 112.98 & 332.84 & 303.96 & 624.7 \\
Intensidad de capital & 73.85 & 109.71 & 247.78 & 146.38 & 98.2 \\
Rentabilidad de fuerza de trabajo & 1.88 & 4.25 & 6.23 & 6.10 & 224.5 \\
\hline
\end{tabular}

Nota: Cifras en miles de pesos de 1973. En el Censo Industrial de 1980 no se presenta información sobre activos fijos, el indicador para dicho año se construyó con datos de 1975.

Fuente: SPP, X Censo Industrial, 1976. INEGI, XI Censo Industrial, 1981. INEGI, XIII Censo Industrial, 1989. INEGI, XV Censo Industrial, 1999. INEGI, XVI Censo Industrial, 2004.

El movimiento de los indicadores comentados se refleja en una tendencia positiva en la evolución de la rentabilidad, y aunque no se incrementa en los últimos años, denota un proceso virtuoso de reestructuración que ha incluido crecimiento, generación de empleo, introducción de procesos técnicos más eficientes y mejoramiento en la remuneración al personal ocupado, lo que implica que hay una retribución a los trabajadores por su contribución en el incremento de la productividad y de la ganancia para el capital.

Productos de minerales no metálicos

La producción de bienes elaborados con minerales no metálicos tuvo un periodo de crecimiento en su producción y empleo en los años ochenta de cerca de $7 \%$ anual en 
promedio. Después entre 1988 y 2003 el crecimiento en la producción se reduce a $1.6 \%$ anual y el empleo cae a una tasa de $-3.4 \%$ en el mismo periodo.

La remuneración media de esta rama se comporta a la baja desde 1980, lo que en términos reales significó una pérdida de poder adquisitivo de sus trabajadores de $47 \%$ a lo largo del periodo. Por otro lado, es notable el constante aumento de la productividad laboral, lo que le ha permitido ubicarse como una de las más consistentes de la región; igualmente importante ha sido el incremento en la intensidad de capital, de tal manera que en 2003 fue la actividad con el índice más elevado en este aspecto. Parte de la explicación se encuentra en la reestructuración y el establecimiento de empresas destinadas a la elaboración de cemento con los más altos niveles tecnológicos como es el caso de Holcim Apasco, así como de la fuerte reestructuración de empresas de capital nacional como Vitromex, que en los años noventa moderniza su producción con procesos productivos flexibles que incluye la utilización de robots. Cabe mencionar que en la primera de las empresas señaladas la presencia de la fuerza de trabajo es mínima y en la segunda la reestructuración implicó una fuerte reducción de la plantilla laboral (cuadro 5).

Cuadro 5

Productos de minerales no metálicos Indicadores de desempeño (1980, 1988, 1998 y 2003)

\begin{tabular}{lrrrrc}
\hline & 1980 & 1988 & 1998 & 2003 & Variación 1980-2003 \\
\hline Remuneración media & 37.38 & 23.61 & 17.21 & 19.77 & $(47.1)$ \\
Productividad laboral & 70.90 & 128.72 & 172.29 & 271.57 & 283.0 \\
Intensidad de capital & 111.30 & 177.03 & 366.68 & 606.62 & 445.0 \\
Rentabilidad de fuerza de trabajo & 1.90 & 5.45 & 10.01 & 13.73 & 622.6 \\
\hline
\end{tabular}

Nota: Cifras en miles de pesos de 1973. En el Censo Industrial de 1980 no se presenta información sobre activos fijos, el indicador para dicho año se construyó con datos de 1975.

Fuente: SPP, X Censo Industrial, 1976. INEGI, XI Censo Industrial, 1981. INEGI, XIII Censo Industrial, 1989. INEGI, XV Censo Industrial, 1999. INEGI, XVI Censo Industrial, 2004.

Debido al aumento en productividad asociado a procesos más intensivos en capital y la tendencia descendente de la remuneración promedio de la rama, la elaboración de productos de minerales no metálicos muestra un sostenido aumento en la rentabilidad del uso de fuerza de trabajo, para colocarse en 2003 con el indicador más alto de la región en este aspecto y casi tres veces el promedio regional.

Esta rama es un ejemplo de reestructuración basada en fuertes inversiones en tecnología y medios de producción, pero con fuerte afectación al trabajo al limitar su 
capacidad generadora de empleo, además de que el trabajador promedio no obtiene una adecuada retribución por su productividad, no obstante la alta rentabilidad que ello significa.

\section{Reestructuración no lograda}

Denominamos actividades de reestructuración no lograda a la industria textil y a la productora de bienes eléctricos y electrónicos, ya que en algún momento del periodo de estudio mostraron intentos por mejorar su lugar en la región, o bien mejoraron en los indicadores de desempeño que, sin embargo, no lograron mantener ni el crecimiento ni la operación bajo condiciones favorables. También en el caso de maquinaria y equipo eléctrico y electrónico, el principal motivo por el que lo ubicamos en esta clasificación es porque el porcentaje de participación en la estructura manufacturera no ha sido tan significativo a pesar de sus posibilidades de articulación con la rama de equipo de transporte, además de que a lo largo del periodo ha mantenido bajos indicadores de intensidad de capital, lo que denota la escasa inversión en medios de producción en relación con el crecimiento mostrado.

Industria textil

Con la transición al modelo de apertura internacional esta actividad enfrentó dificultades en los años ochenta, por lo que prácticamente no creció. Aunque muestra alguna recuperación en los noventa, influye la desaceleración de la economía de Estados Unidos en los primeros años del siglo actual y la mayor presencia en el mercado estadounidense de la producción textil proveniente de países orientales (CEESPAC, 2001). Por lo anterior, su producción y empleo en la región cayeron $1 \%$ y $7 \%$ respectivamente entre 1988 y 2003. La presencia de esta industria en la región propició el arribo de empresas dedicadas a la confección de ropa, aunque estas actividades tienen una presencia mucho más importante en la región de la Laguna (Van Doren, 2003).

La remuneración promedio en términos reales de la industria textil sufrió una reducción de $15 \%$ en los años del ajuste estructural y se ha mantenido sin cambios sustanciales desde entonces, en niveles cercanos a la media regional (cuadro 6).

A pesar de la pérdida de presencia y las tasas negativas de crecimiento que mostró la rama en los años ochenta, su productividad laboral se incrementó. Por otro lado, después de la descapitalización que sufre en esos años, el indicador de intensidad de capital se recupera de manera importante en los años posteriores a 1988. Lo que muestra los esfuerzos por recuperar el crecimiento y mejorar la productividad con métodos de pro- 
ducción más automatizados sustentados en medios de producción flexibles, así como la introducción de conceptos de organización productiva asociados a la producción posfordista como son programas de calidad total, justo a tiempo y trabajo en equipo.

Cuadro 6

Industria textil

Indicadores de desempeño (1980, 1988, 1998 y 2003)

\begin{tabular}{lrrrrc}
\hline & 1980 & 1988 & 1998 & 2003 & Variación 7980-2003 \\
\hline Remuneración media & 30.24 & 25.85 & 27.45 & 27.99 & $(7.4)$ \\
Productividad laboral & 60.73 & 88.84 & 108.89 & 42.94 & $(29.3)$ \\
Intensidad de capital & 78.04 & 27.40 & 236.58 & 192.26 & 146.4 \\
Rentabilidad de fuerza de trabajo & 2.01 & 3.44 & 3.97 & 1.53 & $(23.9)$ \\
\hline
\end{tabular}

Nota: Cifras en miles de pesos de 1973. En el Censo Industrial de 1980 no se presenta información sobre activos fijos, el indicador para dicho año se construyó con datos de 1975.

Fuente: SPP, X Censo Industrial, 1976. INEGI, XI Censo Industrial, 1981. INEGI, XIII Censo Industrial, 1989. INEGI, XV Censo Industrial, 1999. INEGI, XVI Censo Industrial, 2004.

Sin embargo, en los años más recientes el indicador de productividad se reduce sustancialmente, debido a la disminución del precio de productos textiles en el mercado mundial, lo que erosiona la rentabilidad.

Por lo anterior, podemos señalar que la industria textil es ejemplo de un intento de reestructuración, que en los años noventa realizó importantes inversiones que le permiten crecer y aumentar su productividad, pero las condiciones del mercado mundial que se presentan en los primeros años del siglo actual establecen parámetros de competitividad que no puede enfrentar de manera satisfactoria.

\section{Maquinaria y equipo eléctrico y electrónico}

La industria productora de maquinaria y equipo eléctrico y electrónico se asocia a la producción industrial bajo el esquema de maquiladora. Esta actividad parecía haber encontrado en la conformación de una región especializada en la industria automotriz una opción para su desarrollo. Mostró tasas de crecimiento importantes a raíz de la apertura económica, lo que le permitió arribar a una participación en la producción de $3.2 \%$ y de casi $15 \%$ del empleo en 1998, al ubicarse como la segunda rama en importancia en este indicador, sólo superada por equipo de transporte.

Sin embargo, los años que corresponden a la desaceleración del crecimiento de la economía de Estados Unidos son difíciles para esta actividad, por lo que cae a tasas 
promedio anual de alrededor de $13 \%$ en la producción y de $19 \%$ en el empleo, lo que manifiesta el carácter volátil de un segmento de las empresas que la conforman.

La rama ha mantenido niveles de remuneración por debajo de la media regional desde los registros censales de 1980 hasta los de 1998. En esta actividad se manifiesta una correspondencia entre las fluctuaciones de la productividad y la remuneración, ya que avanzan paralelamente en sus altibajos a lo largo del periodo (cuadro 7).

Las fluctuaciones en remuneración media pueden interpretarse como una manifestación de la flexibilidad de la relación salarial, para ajustar la operación de las empresas de la rama a los cambios en el entorno económico, lo que es característico bajo estos esquemas de relocalización. Es probable que el aumento en la remuneración en los años recientes obedezca a que la salida de empresas del mercado se presenta en aquellas relativamente volátiles, mientras que permanecen las más estables y que ofrecen condiciones salariales más favorables.

Cuadro 7

Maquinaria y equipo eléctrico y electrónico

Indicadores de desempeño (1980, 1988, 1998 y 2003)

\begin{tabular}{lrrrrc}
\hline & 1980 & 1988 & 1998 & 2003 & Variación 1980-2003 \\
\hline Remuneración media & 16.54 & 22.10 & 17.75 & 38.78 & 134.5 \\
Productividad laboral & 22.42 & 51.80 & 38.25 & 41.59 & 85.5 \\
Intensidad de capital & 83.08 & 72.29 & 33.73 & 69.78 & $(16.0)$ \\
Rentabilidad de fuerza de trabajo & 1.35 & 2.34 & 2.15 & 1.59 & 17.8 \\
\hline
\end{tabular}

Nota: Cifras en miles de pesos de 1973. En el Censo Industrial de 1980 no se presenta información sobre activos fijos, el indicador para dicho año se construyó con datos de 1975.

Fuente: SPP, X Censo Industrial, 1976. INEGI, XI Censo Industrial, 1981. INEGI, XIII Censo Industrial, 1989. INEGI, XV Censo Industrial, 1999. INEGI, XVI Censo Industrial, 2004.

Si bien esta actividad nunca ha sido de alta productividad ni intensiva en capital, lo largo del periodo 1980-1998 muestra una tendencia hacia procesos cada vez menos intensivos en capital que, sin embargo, logra mantener una evolución positiva de dicha productividad. Lo anterior indica una estrategia de las empresas de enfrentar las circunstancias adversas del mercado con la búsqueda de una mayor intensidad en el uso de la fuerza de trabajo.

La rentabilidad en la rama de maquinaria y equipo eléctrico y electrónico, aunque se deteriora entre 1998 y 2003, mantiene una tendencia positiva a lo largo del periodo. 
Desmantelamiento de la industria metalmecánica

Entre las ramas de actividad manufacturera en la región sureste de Coahuila que han presentado una franca decadencia bajo el modelo de apertura internacional encontramos la metálica básica, productos metálicos, así como maquinaria y equipo.

Las actividades mencionadas, integrantes de la industria metalmecánica, representaban bajo el modelo de economía cerrada el principal núcleo productivo en la región, con 53\% del producto y 49\% del empleo en 1980. A pesar de la especialización regional alcanzada, la transición al modelo de apertura disminuyó drásticamente la presencia de estas ramas, hasta representar de manera conjunta en 2003 sólo 3.6\% del producto y $16 \%$ del empleo. Si bien esta caída es particularmente observable en productos metálicos y en maquinaria y equipo, incluimos en este grupo a metálica básica, por la relación que guarda con las anteriores en la posibilidad de concretar encadenamientos productivos que pudieran articular sus dinámicas.

Llama la atención que con la irrupción del modelo de apertura internacional las actividades comentadas en este bloque entran en un proceso de desindustrialización del núcleo productivo más importante hasta antes de la apertura económica, y no logran integrarse a un crecimiento vigoroso mediante encadenamientos con la rama de equipo de transporte.

Productos metálicos

Con la apertura económica la rama de productos metálicos presentó una caída acelerada en su participación en el sector manufacturero de la región. Desde los años del ajuste estructural la producción y el empleo en la rama descendieron $13 \%$ y $7 \%$ en cada caso, de 1988 a 2003 la caída en producción continúa ahora a un ritmo de casi el $2 \%$ y el empleo aumenta $0.7 \%$ anual en promedio.

Además de ser la actividad con mayor importancia en el producto manufacturero regional en 1980, productos metálicos se significaron en ese año por mostrar una remuneración media superior al promedio de la región. Con el modelo de apertura internacional, la crisis que acusa la rama tuvo un impacto severo en los salarios, al grado que en los últimos años es una de las actividades con remuneración más baja.

Durante todo el periodo de estudio la rama muestra una tendencia a la reducción de la productividad laboral, que en los años ochenta fue acompañada por un aumento importante en la intensidad de capital, resultante de una severa reducción en la plantilla laboral. Con lo anterior se reactivó la rama durante los noventa pero no logra restituir la productividad (cuadro 8).

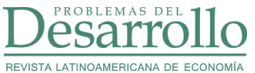


Cuadro 8

Productos metálicos

Indicadores de desempeño (1980, 1988, 1998 y 2003)

\begin{tabular}{lccccc}
\hline & 1980 & 1988 & 1998 & 2003 & Variación 7980-2003 \\
\hline Remuneración media & 37.84 & 15.48 & 18.74 & 16.38 & $(56.7)$ \\
Productividad laboral & 79.85 & 50.35 & 44.75 & 28.71 & $(64.0)$ \\
Intensidad de capital & 39.03 & 120.82 & 53.69 & 63.45 & 62.6 \\
Rentabilidad de fuerza de trabajo & 2.11 & 3.25 & 2.39 & 2.12 & 0.5 \\
\hline
\end{tabular}

Nota: Cifras en miles de pesos de 1973. En el Censo Industrial de 1980 no se presenta información sobre activos fijos, el indicador para dicho año se construyó con datos de 1975.

Fuente: SPP, X Censo Industrial, 1976. INEGI, XI Censo Industrial, 1981. INEGI, XIII Censo Industrial, 1989. INEGI, XV Censo Industrial, 1999. INEGI, XVI Censo Industrial, 2004.

Productos metálicos modificó muy poco su nivel de rentabilidad laboral; aunque el indicador sube entre 1980 y 1988, vuelve a caer para ubicarse en 2003 en un nivel similar al de 1980, lo que resulta contradictorio en un entorno que a partir de la emergencia del modelo de apertura internacional abrió mejores condiciones de rentabilidad para la industria de la región y es una manifestación de que en esta actividad no se realizaron las transformaciones productivas encaminadas a una reestructuración que permitiera mejores condiciones de competitividad, sobre la base de tecnologías de producción modernas, lo que además ha limitado su acceso al mercado externo.

\section{Maquinaria y equipo}

Algo similar a lo ocurrido con productos metálicos se presenta en la rama de maquinaria y equipo, ya que lejos de integrarse a la dinámica económica regional, desde los años ochenta resintió efectos negativos por el cambio de modelo, por lo que cae su producción en $0.7 \%$ y el empleo en $2.7 \%$ en un entorno regional de acelerado crecimiento industrial. Posteriormente entre 1988 y 2003 la caída en su producción es más acentuada a un ritmo de $3 \%$ anual en promedio, aunque revierte la caída en el empleo para crecer $1.6 \%$ promedio anual.

En el caso de la remuneración promedio al personal ocupado, en 1980 registró el indicador más alto de la región con $30 \%$ arriba del promedio. Sin embargo, el ajuste estructural de los años ochenta repercutió severamente en los niveles salariales. Desde esos años junto a la caída en el nivel de actividad y la remuneración media, esta rama vio disminuir sus indicadores de productividad e intensidad de capital. En 
remuneración media e intensidad de capital se recuperó durante los noventa y los primeros años del siglo XXI, pero le será difícil mantener la tendencia si no hay una recuperación también en productividad. En el caso particular de la intensidad de capital, el indicador aumenta básicamente por la contracción en el empleo de casi 10\% promedio anual entre 1998 y 2003, y no por un cambio tecnológico (cuadro 9).

La reducción en rentabilidad que presenta la rama en los últimos años puede inhibir los incentivos para la inversión, a menos que el mejoramiento en los niveles salariales anuncie una reestructuración de los procesos productivos que repercuta de manera favorable en los renglones de productividad.

Cuadro 9

Maquinaria y equipo

Indicadores de desempeño (1980, 1988, 1998 y 2003)

\begin{tabular}{lccccc}
\hline & 1980 & 1988 & 1998 & 2003 & Variación 1980-2003 \\
\hline Remuneración media & 44.44 & 17.91 & 27.07 & 37.36 & $(15.9)$ \\
Productividad laboral & 61.23 & 52.82 & 131.68 & 96.09 & 56.9 \\
Intensidad de capital & 82.01 & 54.68 & 70.60 & 90.64 & 10.5 \\
Rentabilidad de fuerza de trabajo & 1.38 & 2.95 & 4.86 & 2.57 & 86.2 \\
\hline
\end{tabular}

Nota: Cifras en miles de pesos de 1973. En el Censo Industrial de 1980 no se presenta información sobre activos fijos, el indicador para dicho año se construyó con datos de 1975.

Fuente: SPP, X Censo Industrial, 1976. INEGI, XI Censo Industrial, 1981. INEGI, XIII Censo Industrial, 1989. INEGI, XV Censo Industrial, 1999. INEGI, XVI Censo Industrial, 2004.

Por lo anterior, consideramos a esta rama como característica de una reestructuración no realizada en torno a una actividad que aparentemente tendría grandes posibilidades de crecer bajo del modelo de apertura internacional, por la especialización regional alcanzada antes de la apertura y por la posibilidad de articularse con diversas ramas de actividad, sobre todo con equipo de transporte.

\section{Metálica básica}

Bajo el modelo sustitutivo de importaciones, Coahuila alcanzó la especialización productiva en torno a la industria metálica básica, concentrada en la ciudad de Monclova. De lo anterior cabría esperar que con el cambio de modelo y el traslado a la región sureste del eje de acumulación en la entidad se generaran procesos que permitieran un mayor crecimiento de la actividad, mediante su articulación con la industria metalmecánica en general, sin embargo ello no ha ocurrido.

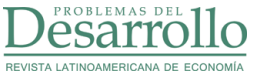


El comportamiento reciente de la remuneración media en la industria metálica básica revirtió una tendencia descendente que había mantenido desde los años ochenta. En lo referente a productividad, muestra un aumento a lo largo del periodo, sobre todo en los años cercanos al cambio de siglo, aunque no debemos olvidar la fuerte reducción en el empleo que contribuye a incrementar este indicador. La rama muestra además una marcada descapitalización en los años noventa, observable en la disminución de la intensidad de capital (cuadro 10).

Cuadro 10

Industria metálica básica

Indicadores de desempeño (1980, 1988, 1998 y 2003)

\begin{tabular}{lrrrrc}
\hline & 1980 & 1988 & 1998 & 2003 & Variación 1980-2003 \\
\hline Remuneración media & 36.75 & 24.78 & 17.30 & 35.38 & $(3.7)$ \\
Productividad laboral & 56.96 & 65.33 & 65.79 & 104.72 & 83.8 \\
Intensidad de capital & 187.41 & 252.15 & 22.39 & 337.53 & 80.1 \\
Rentabilidad de fuerza de trabajo & 1.55 & 2.64 & 3.80 & 2.96 & 91.0 \\
\hline
\end{tabular}

Nota: Cifras en miles de pesos de 1973. En el Censo Industrial de 1980 no se presenta información sobre activos fijos, el indicador para dicho año se construyó con datos de 1975.

Fuente: SPP, X Censo Industrial, 1976. INEGI, XI Censo Industrial, 1981. INEGI, XIII Censo Industrial, 1989. INEGI, XV Censo Industrial, 1999. INEGI, XVI Censo Industrial, 2004.

Entre 1998 y 2003 la rama metálica básica tiene un comportamiento diferente al de la mayoría de las actividades, pues no resiente la desaceleración de la economía estadounidense y aunque parte de una base muy baja, muestra ritmos de crecimiento anual superiores a 50\% tanto en empleo como en producción — aunque partiendo de una base muy baja - con incrementos considerables en productividad y sobre todo en intensidad de capital, lo que es resultado de la realización de inversiones que posiblemente apunten hacia un mayor dinamismo de esta actividad en un futuro.

$\mathrm{El}$ indicador de rentabilidad en la rama metálica básica se incrementó durante el periodo, debido principalmente a la caída en las remuneraciones, ya que no hay aumentos sustanciales en productividad, con excepción de los años recientes en que la capitalización de la rama impulsa su crecimiento, aumenta la intensidad de capital y la productividad, así como la remuneración promedio. Lo anterior repercute en una disminución de la rentabilidad, condición que podría mejorar en la medida que mantenga el crecimiento basado en procesos productivos más tecnificados, más productivos y con fuerza de trabajo más capacitada y mejor remunerada.

Vol. 39, núm. 155, octubre-diciembre / 2008

Dessarrobllo 


\section{Conclusiones}

La transición de México al modelo de apertura internacional implicó un traslado del espacio de acumulación con mayor dinamismo en el aparato manufacturero de Coahuila de la región centro hacia el sureste; paralelamente se modificó la vocación industrial predominante en la entidad de la generación de insumos para sustentar el crecimiento bajo una economía cerrada, hacia la producción de bienes de consumo duradero destinados al mercado externo.

Los procesos de relocalización industrial emprendidos por capitales originarios de economías centrales para restituir la ganancia erosionada por el agotamiento de la base tecnológica e institucional del fordismo, así como las políticas de desregulación aplicadas por el Estado mexicano, encuentran en la región sureste de Coahuila condiciones propicias para la inversión, el caso más representativo se encuentra en la rama de equipo de transporte. Esta actividad se convirtió en el eje en torno al cual se inicia la reestructuración productiva de la región, mediante la introducción de procesos intensivos en capital y basados en la tecnológica de la producción flexible posfordista.

Asimismo, la actividad automotriz se constituyó en el centro de atracción de inversión tanto nacional como extranjera y en principal mecanismo de integración con la economía mundial. Además de equipo de transporte, otras industrias como las de alimentos, química y la elaboración de productos de minerales no metálicos emprenden transformaciones observables en la evolución de indicadores como productividad, intensidad de capital y rentabilidad, que les permiten mantener un lugar en la manufactura de la región. Cada una de ellas bajo diferentes estrategias: la industria de productos de minerales no metálicos y la química lo hacen con fuertes inversiones para automatizar los procesos productivos, basados en medios de producción de alta tecnología integrada a los conceptos de la producción posfordista. Por el contrario, la industria de alimentos emprende una estrategia de crecimiento sustentada en la intensificación del uso de la fuerza de trabajo.

El estudio permitió también distinguir ramas de actividad en las que se observaron intentos por consolidar una reestructuración que finalmente no se concretó, como ocurrió en los casos de la industria textil y la de productos eléctricos y electrónicos, actividades que en los últimos años presentan señales de descapitalización y pérdida de productividad. Es particularmente interesante el caso de la industria textil, ya que intentó una modernización incorporando tecnología y conceptos productivos de la producción flexible, que no consolidaron un proceso sostenido y firme de reposicionamiento en la industria de la región, por factores relacionados con la competencia de 
productos asiáticos en el mercado nacional y en el principal mercado de los productos nacionales de exportación, Estados Unidos.

Por otro lado, las actividades que fueron el núcleo sustancial de la industria en la región antes de la apertura económica representan un proceso acelerado de desindustrialización; las ramas de metálica básica, productos metálicos, así como maquinaria y equipo son actividades que no logran integrarse a la dinámica de crecimiento manufacturero de la región ni se articulan al crecimiento de la industria automotriz; en éstas el desempeño de la productividad, la inyección de capital y los niveles de rentabilidad han sido pobres.

Por lo anterior, la transformación estructural de la manufactura en el sureste de Coahuila bajo el modelo de economía abierta es el resultado de la confluencia de factores como la especialización alcanzada antes de la apertura, la posibilidad de atraer inversión capaz de inyectar capital para la aplicación de procesos productivos acordes al régimen posfordista de acumulación, así como integrarse al mercado externo mediante la exportación.

Aunque no pueden soslayarse los beneficios del crecimiento, sobre todo si consideramos las condiciones que ha enfrentado el país, es pertinente señalar que una vez consolidado el modelo de apertura internacional en nuestro país, la industria manufacturera en la región ha reducido su capacidad para generar empleos. Asimismo, la evolución industrial ha dado lugar a una fuerte concentración de la actividad en torno a la industria automotriz, lo que hace depender demasiado a la región del capital y el mercado estadounidense, y hace vulnerable a la región, sobre todo en fases recesivas de la economía de aquel país.

Vol. 39, núm. 155, octubre-diciembre / 2008 
Bibliografía

Aboites, Jaime, Industrialización y desarrollo agrícola en México, México, UAM-X Plaza y Valdés, 1989.

Abramo, Laís, "Mercado de trabajo, flexibilización y nuevas formas de regulación", en Trabajo, núm. 4, UAM, julio de 2001. pp. 160-183.

Aglietta, Michel, Regulación y crisis del capitalismo, México, Siglo XXI, 1979.

Arteaga, Arnulfo, "Reconversión industrial y flexibilidad del trabajo en la industria automotriz en México, 1981-1986", en Esthela Gutiérrez Garza (coord.) Testimonios de la crisis 3. Austeridad y reconversión, México, Siglo XXI- UNAM, 1988, pp. 166-187.

"Innovación tecnológica y clase obrera en la industria automotriz", en Esthela Gutiérrez Garza (coord.), Testimonios de la crisis 1. Reestructuración productiva y clase obrera, México, Siglo XXI-UNAM, 1985, pp. 146-169.

Basave, Jorge, "Modalidades de integración internacional y perspectivas de expansión de empresas mexicanas", en Jorge Basave, Alejandro Dabat, Carlos Morera, Miguel A. Ríos y Francisco Rodríguez (coord.), Globalización y alternativas incluyentes para el siglo XXI, México, UNAM, 2002, pp. 435-460.

Calderón, Cuauhtémoc y Gerardo Martínez, "Estructura industrial de la frontera norte y estrategia de desarrollo" en Comercio Exterior, vol. 54, núm 8, México, Banco Nacional de Comercio Exterior, agosto de 2004, pp. 712-720.

Cárdenas, Fe Esperanza y Redonnet, Vicente, "El desempleo en la empresa Altos Hornos de México S. A. En la década de los noventa", en Cuadernos de Investigación, Área III, núm. 18, Saltillo, Universidad Autónoma de Coahuila, 1998, pp. 7-29.

Centro de Estudios Económicos del Sector Privado, A. C. La entrada de China a la OMC: retos y oportunidades para México, México, 2001.

Coriat, Benjamín, El taller y el robot, México, Siglo XXI, 1992.

, Pensar al revés. Trabajo y organización en la empresa japonesa, Madrid, Siglo XXI, 1992.

Dávila, Alejandro, "Nuevas tendencias de la subcontratación internacional en México.
El caso de la industria manufacturera del noreste", en Antonio Argüelles y J. Antonio Gómez Mandujano (comp.), La competitividad de la industria mexicana frente a la concurrencia internacional, México, FCENAFIN, 1994, pp. 168-190.

, Los agrupamientos económicos de su sector industrial, Saltillo, Gobierno de Coahuila, s/f.

Dávila, Mario, Análisis del sector exportador de Coahuila, Saltillo, Universidad Autónoma de Coahuila, 1998.

El complejo automotriz de Ramos Arizpe, Coahuila, Saltillo, Universidad Autónoma de Coahuila, 1982.

De Bell, Leendert, Globalization, regional development and local response, Amsterdam, Dutch University Press, 2005.

Gutiérrez, Elizabeth, "El patrón de distribución regional de la industria en Coahuila 19601985”, en Cuadernos de Investigación, núm. 10, Saltillo, Universidad Autónoma de Coahuila, 1993, pp. 223-336.

Gutiérrez Garza, Esthela, "Impacto del TLCAN en las relaciones del trabajo en México", Conferencia ofrecida en el curso: diálogo social, globalización e integración regional, OIT-UANL, Monterrey, mimeo, octubre de 2004.

, Teorías del desarrollo en América Latina, México, Trillas, 2003.

INEGI, XI Censo Industrial, 1981, México, 1988 .

XIII Censo Industrial, Coahuila. 1989, Aguascalientes, 1992.

XV Censo Industria, 1999, Aguascalientes, 2001.

, XVI Censo Industrial, 2004, Aguascalientes, 2005.

Leborgne, Danielle y Alain Lipietz, “Ideas falsas y cuestiones abiertas sobre el posfordismo" en Trabajo, núm. 8, México, UAM-I, 1992.

Lipietz, Alain, "El mundo del posfordismo" en Ensayos de Economía, vol. 7, núm. 12, Medellín, 1997.

Mariña, Abelardo, "Balance y perspectivas de la industria manufacturera mexicana tras veinte años de reestructuración neoliberal: integración subordinada a Estados Unidos, desindustrialización y precarización del empleo", trabajo presentado en IX Jornadas 
de Economía Crítica, Universidad Complutense de Madrid, 2004.

Martínez, Enrique, Sexto informe de gobierno. Anexo Estadístico, Saltillo, Gobierno del Estado de Coahuila, 2005.

Martínez, Gerardo, "Estructura de la industria maquiladora de exportación y su grado de integración regional", en Cuadernos de Investigación, núm. 10, Saltillo, Universidad Autónoma de Coahuila, 1993.

Mendoza, J. Eduardo, "Crecimiento y especialización en la región Saltillo-Ramos Arizpe", en Comercio Exterior, vol. 51, núm 3, México, marzo de 2001, pp. 250-258.

Meléndez, Adriana, El impacto del crecimiento industrial y urbano sobre el subsector ejidal de Monclova, Coahuila, tesis de maestría en Planeación Agropecuaria, Saltillo, Universidad Autónoma Agraria Antonio Narro, 1994

Micheli, Jordy, Nueva manufactura, globalización y producción de automóviles en México, UNAM, México, 1994.

Ramírez, José Carlos, "Los nuevos factores de localización industrial en México. La experiencia de los complejos automotrices de exportación en el norte de México" en Economía Mexicana, nueva época, vol. VIII, núm. 1, México, CIDE, primer semestre de 1999.
Rendón, Teresa, "Tendencias del empleo en México", en Comercio Exterior, México, vol. 49, núm. 5, mayo de 1999, pp. 251-259.

Schonberger, Richard, Técnicas japonesas de fabricación, México, Limusa, 1987.

Soria, Víctor, Crecimiento económico, crisis estructural y evolución de la pobreza en México, México, UAM-Plaza y Valdés, 2000.

Sotelo, Adrián, "Flexibilidad regresiva y tendencias del trabajo en la mundialización del capital", en Trabajadores, núm 30, México, Universidad Obrera de México, mayo-junio de 2002, pp. 32-35.

"Política y reconversión industrial en México", en Gutiérrez, Esthela (coord.), Testimonios de la crisis, núm. 3. Austeridad y Reconversión, México, Siglo XXI-UNAM, 1988, pp. 188-208.

Valenzuela, José Carlos, "La reconversión industrial en el contexto del nuevo patrón de acumulación secundario-exportador", en Gutiérrez, Esthela (coord.), Testimonios de la crisis, núm. 3. Austeridad y Reconversión, México, Siglo XXI-UNAM, 1988, pp. 110-165.

Van Dooren, Robine, Garments on the move: The local dynamics of export networks in La Laguna, México, Amsterdam, Rozemberg Publishers, 2003. 